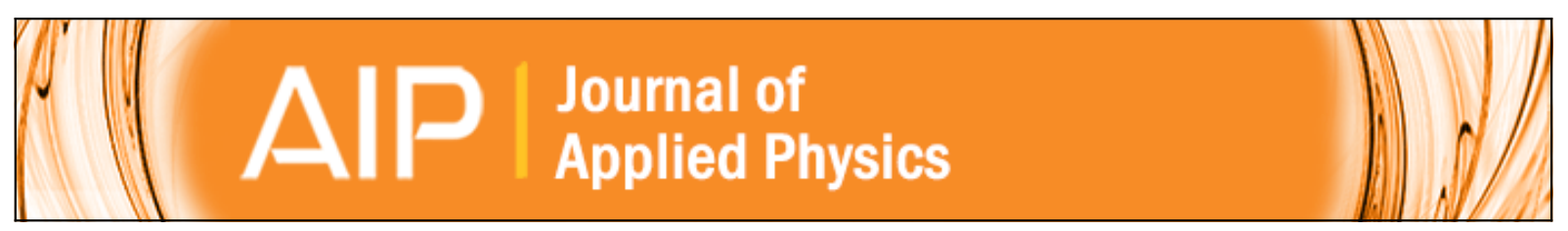

\title{
A dipole lattice model of switching characteristics in ferroelectric superlattices
}

K.-H. Chew, Y. Zhou, L.-H. Ong, and M. Iwata

Citation: Journal of Applied Physics 114, 224108 (2013); doi: 10.1063/1.4846797

View online: http://dx.doi.org/10.1063/1.4846797

View Table of Contents: http://scitation.aip.org/content/aip/journal/jap/114/22?ver=pdfcov

Published by the AIP Publishing

\section{Articles you may be interested in}

Dipole spring ferroelectrics in superlattice $\mathrm{SrTiO} / \mathrm{BaTiO} 3$ thin films exhibiting constricted hysteresis loops

Appl. Phys. Lett. 100, 092905 (2012); 10.1063/1.3691172

Modeling the switching kinetics in ferroelectrics

J. Appl. Phys. 110, 114106 (2011); 10.1063/1.3660680

Modeling ferroelectric capacitors based on the dipole switching theory

J. Appl. Phys. 101, 104505 (2007); 10.1063/1.2729470

Growth, microstructure, and ferroelectric properties of $\mathrm{Pb}(\mathrm{Zr} 0.4 \mathrm{Ti} 0.6$ ) $\mathrm{O} 3 \mathrm{~Pb} \mathrm{Zr} O 3$ superlattices prepared on $\mathrm{Sr} \mathrm{Ti} O 3$ (100) substrates by pulsed laser deposition

J. Appl. Phys. 101, 054118 (2007); 10.1063/1.2710285

Hysteresis loops of ferroelectric bilayers and superlattices

Appl. Phys. Lett. 77, 2755 (2000); 10.1063/1.1320040

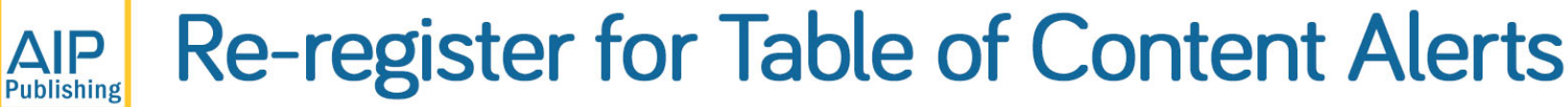




\title{
A dipole lattice model of switching characteristics in ferroelectric superlattices
}

\author{
K.-H. Chew, ${ }^{1, a)}$ Y. Zhou, ${ }^{2,3}$ L.-H. Ong, ${ }^{4}$ and M. Iwata ${ }^{5}$ \\ ${ }^{1}$ Center for Theoretical and Computational Physics, Department of Physics, University of Malaya, \\ 50603 Kuala Lumpur, Malaysia \\ ${ }^{2}$ Department of Physics, University of Hong Kong, Hong Kong, People's Republic of China \\ ${ }^{3}$ Center of Theoretical and Computational Physics, University of Hong Kong, Hong Kong, People's Republic of China \\ ${ }^{4}$ School of Physics, Universiti Sains Malaysia, 11800 Minden, Penang, Malaysia \\ ${ }^{5}$ Department of Engineering Physics, Electronics and Mechanics, Graduate School of Engineering, \\ Nagoya Institute of Technology, Nagoya 466-8555, Japan
}

(Received 20 September 2013; accepted 27 November 2013; published online 12 December 2013; publisher error corrected 28 January 2014)

\begin{abstract}
Using a one-dimensional lattice model, we studied the switching characteristics of superlattices formed out of alternate ferroelectric and dielectric layers. The two layers interact with each other through the dipole lattices located at interface. Switching characteristics of pulsed field such as hysteresis loop, differential electric susceptibility as a function of applied field, and switching current as a function of applied field were examined. Influence of various model parameters included field amplitude, viscosity, interaction between dipole lattices at interface, interaction between dipole lattices within the layer, layer thickness and dielectric stiffness on the switching characteristics were investigated in detail. (C) 2013 AIP Publishing LLC. [http://dx.doi.org/10.1063/1.4846797]
\end{abstract}

\section{INTRODUCTION}

Ferroelectric superlattices are currently a topic of active research ${ }^{1}$ because of their potential applications in ferroelectric memories, ${ }^{2}$ as well as fundamental interest. ${ }^{3}$ Experimental studies on ferroelectric superlattices include $\mathrm{KTaO}_{3} / \mathrm{KNbO}_{3},{ }^{4,5} \mathrm{BaTiO}_{3} / \mathrm{SrTiO}_{3},{ }^{6,7} \mathrm{BaTiO}_{3} /(\mathrm{Ba}, \mathrm{Sr}) \mathrm{TiO}_{3},{ }^{8}$ $\mathrm{BiFeO}_{3} / \mathrm{SrTiO}_{3},{ }^{9}$ and $\mathrm{PbTiO}_{3} / \mathrm{SrTiO}_{3} .{ }^{10}$ While the polarization switching in ferroelectric is a topic of interest, the switching mechanism remains to be a classical unanswered problem for many decades. When dealing with a ferroelectric superlattice system, intermixed layers may form at interfaces between two ferroelectrics. ${ }^{11-15}$ These interface intermixed layers with properties different from those of the constituent layers may affect the properties of the superlattice structure. The formation of intermixed layers can be induced by short-range interactions between materials in-contact, surface or interface reconstruction, cation intermixing or composition deviations at the interfaces in superlattices of ferroelectric solid solution. ${ }^{16}$

Many theoretical studies on switching behaviors of ferroelectric superlattices were performed using the Landau-type theory. ${ }^{17-21}$ A model based on Landau theory is developed to study the hysteresis loop behaviors of ferroelectric superlattices with antiferroelectric interlayer coupling. ${ }^{17,18}$ Several authors extend the model by taking into account the spatial variation of polarization with each constituent layers using the Landau-Ginzburg theory. ${ }^{18-20}$ By treating the ferroelectrics as wide band-gap semiconductors, Liu and Li studied the effect of space charges on the hysteresis loops of ferroelectric superlattices based on Landau-Ginzburg theory. ${ }^{22}$ Those works, ${ }^{17-22}$ however, do not consider interface intermixing in

\footnotetext{
a) Author to whom correspondence should be addressed. Electronic mail: khchew@um.edu.my
}

ferroelectric superlattices. Pertsev and Tyunina ${ }^{16}$ investigated the dielectric properties of superlattice by introducing an interface layer with properties different from those of the constituent layers. However, the model is only applicable to system with layers relatively thick compared to the correlation length of ferroelectricity. The work does not consider the effect of surface or interface; thus, the polarization is homogenous without the local polarization coupling at the interface.

Based on the lattice dipole model, ${ }^{23-27}$ we have proposed to study the influence of interface on the polarization and dielectric properties of a superlattice consisting of alternating ferroelectric and dielectric layers. ${ }^{28}$ However, the switching behavior of ferroelectric superlattices was not discussed. We have shown that the lattice model of ferroelectric superlattices ${ }^{28}$ can be casted into a continuum LandauGinzburg theory. ${ }^{29,30}$ In the model, the interface energy can be interpreted as the formation of interface "dead" layer that are mutually coupled though polarization. ${ }^{29-31}$ In particular, the interface parameter describes the interface intermixing in superlattices. ${ }^{29-31}$ Explicit expressions derived from the interface structure shows that the intermixed layer is determining by the physical properties of the two layers. ${ }^{32,33}$ Using a Landau-Ginzburg theory, it is shown that the interface intermixing leads to a reduction in the coercive field, though the associated remnant polarization is strongly enhanced by a soft dielectric layer. ${ }^{29}$ The switching dynamics of the superlattice are found to be significantly dependent on interface intermixing, layer thickness and the dielectric softness of dielectric layer based on the Landau-Khalatnikov model. ${ }^{34}$ The hysteresis loop behavior of ferroelectric superlattices was studied by taking into account the timedependent space-charge-limited conductivity. ${ }^{35}$

In this work, we investigate the switching characteristics of a superlattice composed alternating ferroelectric and dielectric layers driven by a pulsed electric field. Based on a 
dipole lattice model of ferroelectric superlattice, ${ }^{28}$ the switching characteristics of the superlattice is examined by looking at the hysteresis loop, differential dielectric susceptibility versus applied field and switching current versus applied field. The parameters that govern the switching characteristics of a superlattice consisting of alternate layer of ferroelectric and dielectric will be discussed in detail.

\section{A DIPOLE LATTICE MODEL OF FERROELECTRIC SUPERLATTICES}

We consider a periodic superlattice composed of alternate layer of ferroelectric and dielectric, as shown in Fig. 1(a). The two layers interact with each other via the polarizations located at the interfaces. We construct the model using a dipole lattice model, as proposed by Ishibashi. ${ }^{23-27}$ The dipole lattice model has been used to study the polarization reversal in ferroelectric thin films. ${ }^{36-38}$ Within the framework of a dipole lattice model, ${ }^{23-27}$ each layer is an ensemble of dipole lattices characterized by polarization, which has double potential wells. For simplicity, we consider the simple case of one-dimensional ferroelectric superlattice consisting of alternating layer 1 and layer 2 with total number of $M$ and $N$ lattices, respectively, as depicted in Fig. 1(b). $p_{m}$ and $q_{n}$ represent dipole lattices located at the $m$ th and $n$-th sites of layer 1 and layer 2, respectively. Each dipole interacts with its nearest-neighboring dipoles. $\kappa_{1}$ and $\kappa_{2}$ denote the interaction parameter between the nearestneighboring dipoles within layer 1 and layer 2 , respectively.

The free energy for the ferroelectric layer $l$ with total dipole lattices $M$, is ${ }^{28}$

$$
F_{1}=\sum_{m=1}^{M}\left[\frac{\alpha_{1}}{2} p_{m}^{2}+\frac{\beta_{1}}{4} p_{m}^{4}-p_{m} e\right]+\sum_{m=2}^{M}\left[\frac{\kappa_{1}}{2}\left(p_{m}-p_{m-1}\right)^{2}\right],
$$

and the free energy for the dielectric layer 2 with total lattices $N$ is

$$
F_{2}=\sum_{n=1}^{N}\left[\frac{\alpha_{2}}{2} q_{n}^{2}+\frac{\beta_{2}}{4} q_{n}^{4}-q_{n} e\right]+\sum_{n=2}^{N}\left[\frac{\kappa_{2}}{2}\left(q_{n}-q_{n-1}\right)^{2}\right] \text {, }
$$

where the higher order $p$ and $q$ terms are truncated. $e$ denotes the applied electric field. In the ferroelectric phase, $\alpha_{1}<0$ and $\beta_{1}>0$, whereas $\alpha_{2}>0$ and $\beta_{2}>0$ for the paraelectric layer. The dipoles at the interface of layer 1 and layer 2 are given by $p_{1}=p_{M}$ and $q_{1}=q_{N}$, respectively. It is easily seen that the interaction energy between the dipoles at the interface of the two constituent layers is given by ${ }^{28}$

$$
F_{i}=\frac{\lambda}{2}\left[\left(p_{M}-q_{1}\right)^{2}+\left(p_{1}-q_{N}\right)^{2}\right],
$$

where the interaction parameter between the interface dipole lattices is given by $\lambda$. Within the framework of dipole lattice model, ${ }^{23-27} \kappa_{j}(j=1$ or 2$)$ describes the strength of interaction between neighboring atoms (or polarizations) within the same layer. $\lambda$ gives the strength of coupling between the polarization localized at the interface. For ferroelectrics with second order phase transition, the correlation length $K_{j}^{-1}$ characterizing the domain wall thickness is $\sqrt{-\kappa_{1} / \alpha_{1}}$, whereas $\sqrt{\kappa_{2} / \alpha_{2}}$ is the correlation length of the dielectric layer.

It is important to mention here that if the variation of the order parameter within each layer is smooth and each layer consists of a large number of dipoles, the interaction energy of layer 1 (second term in Eq. (1)) can be approximated as follows: ${ }^{28} \sum_{m=2}^{M}\left[\frac{\kappa_{1}}{2}\left(p_{m}-p_{m-1}\right)^{2}\right] \approx \int_{0}^{L_{1}} \frac{\kappa_{1}}{2}\left(\frac{d p}{d x}\right)^{2} d x$, where $L_{1}=M a_{1}$ and $a_{1}$ are the thickness and the lattice constant of layer 1 , respectively. Similarly, the interaction energy of layer 2 with thickness $L_{2}=N a_{2}$ and its lattice constant $a_{2}$ (second term in Eq. (2)) is given by $\sum_{n=2}^{N}\left[\frac{\kappa_{2}}{2}\left(q_{n}-q_{n-1}\right)^{2}\right]$ $\approx \int_{0}^{L_{2}} \frac{k_{2}}{2}\left(\frac{d q}{d x}\right)^{2} d x$, and $L=L_{1}+L_{2}$ is the periodic thickness.

Thus, it is clear that the interface energy has the same form as the interaction energy term of the dipole lattice model, or

(a)

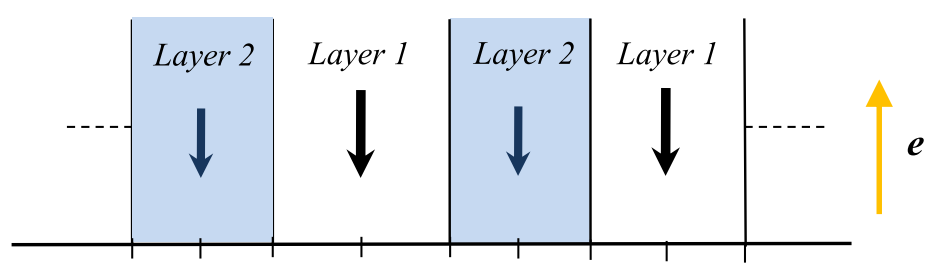

(b)

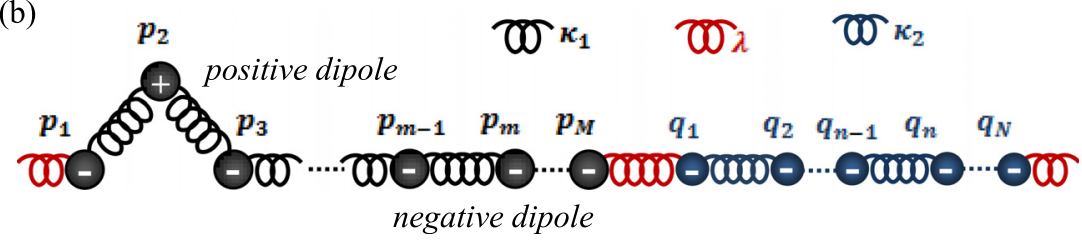

FIG. 1. (a) Schematic illustration of a periodic superlattice composed of alternating ferroelectric layer 1 and dielectric layer 2. Black and blue arrows indicate the direction of polarization for layer 1 and layer 2, respectively. Orange arrow denotes the direction of applied electric field $E$. (b) A dipole lattice model of ferroelectric superlattices composed of alternating layer 1 and layer 2 with total number of $M$ and $N$ lattices, respectively. $p_{m}$ and $q_{m}$ represent dipole lattices, lying in double potential wells, located at the $m$-th and $n$-th sites of layer 1 and layer 2 , respectively. $\kappa_{1}$ and $\kappa_{2}$ describe the interaction parameters between the nearestneighboring dipoles within layer 1 and layer 2 , respectively. At the interface, interaction parameter between the interface dipole lattices is described by $\lambda$. 
the gradient term of the continuum model (the LandauGinzburg theory), which describes the inhomogeneity of polarization within the constituent layer.

Since there are only two dipoles at each interface contributing to the interface coupling energy, the interface energy (3) remains unchanged. By symmetry, the periodic boundary condition gives $p_{M}=p_{1}=p_{i}$ and $q_{N}=q_{1}=q_{i}$, and thus Eq. (3) becomes $F_{i}=\frac{\lambda}{2}\left(p_{i}-q_{i}\right)^{2}=\frac{\lambda}{2}\left(p_{i}^{2}+q_{i}^{2}\right)$ $-\lambda p_{i} q_{i} \cdot{ }^{29-31}$ The former term is analogous to the formation of "dead" layers ${ }^{39-41}$ at the interface region, i.e., the surfaces of layer 1 (" $\lambda p_{i}^{2} / 2$ ") and layer 2 (" $\lambda q_{i}^{2} / 2$ "). The dead layers are linear dielectrics, and their dielectric stiffnesses are determined by the interface parameter $\lambda>0$. The second term " $\lambda p_{i} q_{i}$ " describes the mutual interaction between the local polarization at interfaces (i.e., $p_{i}$ and $q_{i}$ ) due to the modification of bonding at the interfaces, which has the same form as the coupling term (the linear term) in the Landau-like formulation by taking the continuum approximation of transverse Ising model. ${ }^{42}$ In the superlattice system, $\lambda$ describes the intermixing at interface between two layers in continuum model. ${ }^{29-31}$

The interfacial coupling $\lambda \neq 0$ leads to inhomogeneity of polarization near the interfaces. ${ }^{29-31}$ On the other hand, if $\lambda=0$ the polarization is homogenous throughout the constituent layers, regardless of the thickness of layer 1 or layer 2 . In this case, the coercive field of the superlattice $e_{C}$, under an assumption of homogenous electric field $e$, can be obtained from $d e / d p=0$ as

$$
e_{C}= \pm \frac{2 \alpha_{1}}{3}\left(-\frac{\alpha_{1}}{3 \beta_{1}}\right)^{1 / 2}
$$

which is also the coercive field of the ferroelectric layer 1 .

In order to study the process of polarization reversal, we need to take into account the viscosity that causes the delay in motion of the individual polarization. The time-dependent Landau-Ginzburg equations of motion are

$$
\begin{aligned}
\gamma_{1} \frac{d p_{m}}{d t}= & -\frac{\partial F}{\partial p_{m}}=-\left\{\alpha_{1} p_{m}+\beta_{1} p_{m}^{3}\right. \\
& \left.-\kappa_{1}\left(p_{m+1}-2 p_{m}+p_{m+1}\right)-e\right\}
\end{aligned}
$$

and

$$
\gamma_{2} \frac{d q_{n}}{d t}=-\frac{\partial F}{\partial q_{n}}=-\left\{\alpha_{2} q_{n}-\kappa_{2}\left(q_{n+1}-2 q_{n}+q_{n+1}\right)-e\right\},
$$

where $\gamma_{1}$ and $\gamma_{2}$ are the viscosity coefficients for layer 1 and 2 , respectively.

The average polarization of the superlattice is given by

$$
P=\frac{1}{L}\left[\sum_{m=1}^{M} p_{m}+\sum_{n=1}^{N} q_{n}\right]
$$

and the current response is obtained by

$$
i=\frac{1}{L}\left[\sum_{m=1}^{M} \frac{d p_{m}}{d t}+\sum_{n=1}^{N} \frac{d q_{n}}{d t}\right]
$$

In the present study, the switching characteristics of the superlattices are obtained using the applied electric $e$ as

$$
e=e_{0} \sin (2 \pi f t)
$$

where $e_{0}$ and $f$ denote the amplitude and frequency, respectively. In the present study, the simulation is conducted using Eq. (5). Differential electric susceptibility versus applied electric field is obtained by calculating $\mathrm{d} P / \mathrm{d} e$.

\section{RESULTS AND DISCUSSION}

In this section, the calculations are conducted using a relaxation method. ${ }^{43}$ For the convenience of discussion, we denote the superlattice with $M$ and $N$ unit layers of ferroelectric and dielectric, respectively, as "a $M-N$ superlattice." The following parameters are adopted unless otherwise specified: $\alpha_{1}=-1, \alpha_{2}=1, \beta_{1}=\beta_{2}=1, \kappa_{1}=\kappa_{2}=1, \gamma_{1}=\gamma_{2}=1$, and $\lambda^{-1}=0.02$. The correlation lengths correspond to the ferroelectric and dielectric layers are $K_{1}^{-1}=1$ and $K_{2}^{-1}=1$, respectively. For a "5-5" superlattice structure, we have $M=$ $5>2 K_{1}^{-1}$ and $N=5>2 K_{2}^{-1}$. We first examine the switching process by applying a sequence of pulsed electric field to a strongly coupled " $5-5$ " superlattice with $\lambda^{-1}=0.02$, and investigate the switching current $i(t)$ and polarization response $P(t)$, as shown in Fig. 1. The pulsed field is generated using Eq. (8) by taking only pulses with polarity in the direction opposite to the initial polarization. The polarization switching event begins with the initial polarizations in the negative states when $t=0$. The switching process, understandably, depends strongly on the field amplitude $e_{0}$ and pulse length $t_{p}$ or frequency $f$.

Figure 2(a) shows the results of pulse switching with pulse length $t_{p}=50(f=0.01)$ for field amplitudes $e_{0}: 0.2 e_{\mathrm{C}}$ $(-), 0.4 e_{\mathrm{C}}(-)$, and $0.8 e_{\mathrm{C}}(-)$. The polarization remains in negative states when pulses with $e_{0}=0.2 e_{\mathrm{C}}$ is applied. It is seen that the state of polarization changes from negative to positive states when the first pulse with $e_{0}=0.4 e_{\mathrm{C}}(-)$ or $0.8 e_{\mathrm{C}}(-)$ is applied. This is characterized by the maximum value $i_{\max }$ of current response and the time $t_{\max }$ at which it occurs. The switching time $t_{\max }$ for the case with $e_{0}=0.8 e_{\mathrm{C}}$ (-) is shorter than that for the case with $e_{0}=0.4 e_{\mathrm{C}}(-)$, as expected. The decay of polarization after the removal of the applied field is called back-switching, which leads to negative current flow. When the second and third positive pulses are applied in the positive states, we find that the current response is different from that by the first pulse. The coupling at the interface between the constituent layers reduces the coercive field $e_{\mathrm{C}}$ for polarization switching, indicating the presence of interface-aided polarization switching. ${ }^{44}$ In Fig. 2(b), we investigate the switching process produced by different pulse lengths $t_{p}$.

In this study, a series of pulses with field amplitude $e_{0}=0.5 e_{\mathrm{C}}$ is applied and the duration between those pulses is set as 50. It is seen that for certain field amplitude $e_{0}$, there exists a critical pulse length $t_{p}^{*}$ at which the crystal begins to switch. No switching is expected for field pulses shorter than a critical duration $t_{p}<t_{p}^{*}$. If $t_{p}>t_{p}^{*}$, the switching process is completed. We can now study the switching characteristics 

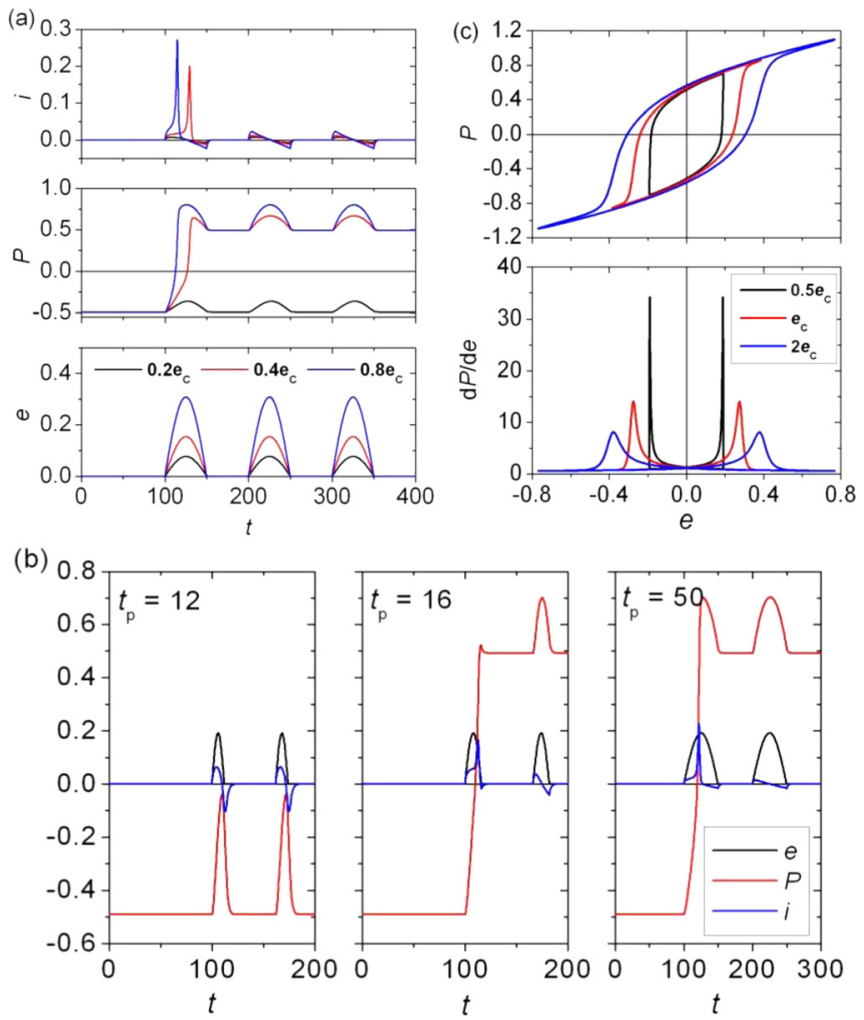

FIG. 2. Pulses of applied field, of polarization and of switching current (a) for different $e_{0}$ with $t_{p}=50$ and (b) for different $t_{p}$ with $e_{0}=0.5 e_{C}$. (c) Applied electric field $e$ dependent of switching characteristics with $e$ : $0.5 e_{C}(-), e_{C}(-)$, and $2 e_{C}(-)$. In the calculations, $\alpha_{1}=-1, \alpha_{2}=1$, $\beta_{1}=\beta_{2}=1, \kappa_{1}=\kappa_{2}=1, \gamma_{1}=\gamma_{2}=1, \lambda^{-1}=0.02$, and $M=N=5$.

of a strongly coupled " $5-5$ " superlattice $\lambda^{-1}=0.02$ produced by bipolar pulses, and it is featured by Eq. (8). Switching characteristic studies include $P$ - $e$ hysteresis loop, differential electric susceptibility as a function of applied field $\mathrm{d} P / \mathrm{d} e-e$ and switching current as a function of applied field $i$-e. In these studies, the frequency of the field is chosen as $f=0.01$ (i.e., pulse length $t_{p}=50$ ). Figure 1(c) shows the field amplitude $e_{0}$ dependent of switching characteristics. It is clearly seen that both the coercive field and polarization increase with increasing field amplitude, which is the well-known experimental results. As $e_{0}$ increases, the maximum value of $\mathrm{d} P / \mathrm{d} e$ reduces and the slope decreases.

Figure 3 shows the viscosity coefficients dependent of switching characteristics for a strongly coupled " $5-5$ " superlattice. $\gamma_{1}$ and $\gamma_{2}$ denote the viscosity coefficients of the ferroelectric and dielectric layers, respectively. The results show that the coercive field is strongly enhanced, as $\gamma_{1}$ or $\gamma_{2}$ decreases. This indicates that the switching time decreases if the value of $\gamma_{1}$ or $\gamma_{2}$ decreases, as shown in Fig. 3(c). The slope of $\mathrm{d} P / \mathrm{d} e-e$ decreases and the maximum value of $\mathrm{d} P / \mathrm{d} e-e$ increases with decreasing the value of $\gamma_{1}$ or $\gamma_{2}$. In comparison with decreasing $\gamma_{1}$, the effect of $\gamma_{2}$ on the coercive field and the slope of $\mathrm{d} P / \mathrm{d} e-e$ is more marked. The polarization is slightly reduced, as $\gamma_{1}$ decreases. Changes in $\gamma_{2}$, however, do not induce an obvious impact on the polarization behavior of the superlattice.

Influence of interface coupling $\lambda$ on the switching characteristics of the superlattices is examined in Fig. 4. Inset
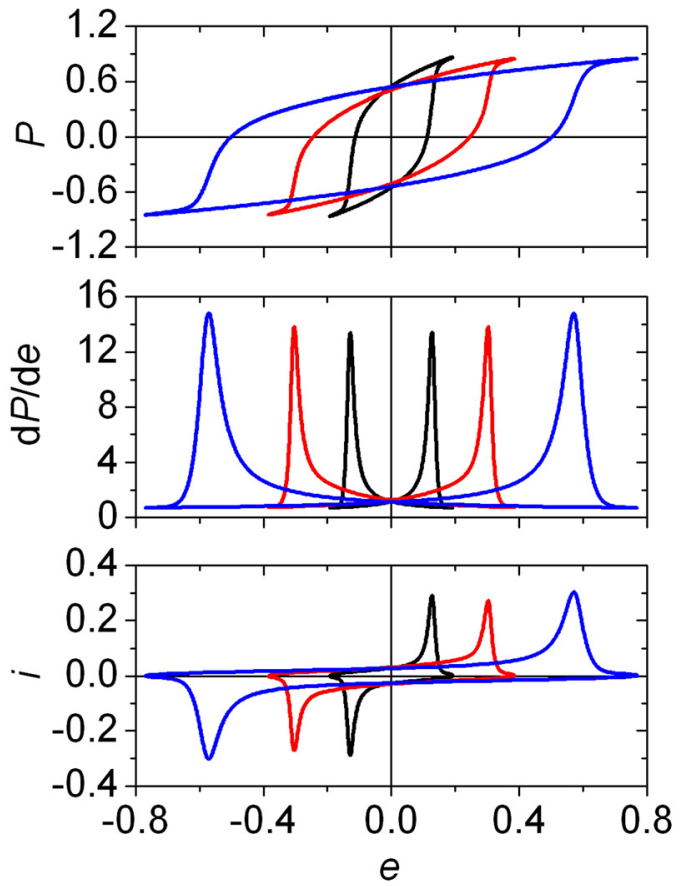

FIG. 3. Influence of viscosity on switching characteristics with $e_{0}=e_{C}$. The values of $\gamma_{1}$ and $\gamma_{2}$ are: 2 and $1(-), 0.5$ and $1(-)$, and 1 and $0.5(-)$, respectively. In the calculations, $\alpha_{1}=-1, \alpha_{2}=1, \beta_{1}=\beta_{2}=1, \kappa_{1}=\kappa_{2}=1$, $\lambda^{-1}=0.02$, and $M=N=5$.

shows the distribution of polarizations in the initial states $t=0$. If $\lambda=0$, polarization remains homogenous throughout the ferroelectric layer, whereas no polarization is induced in the dielectric layer. The existence of the interface coupling leads to a suppression of polarization near the ferroelectric surface, and forms an interface-induced ordered state in the dielectric layer. The suppression of polarization in the
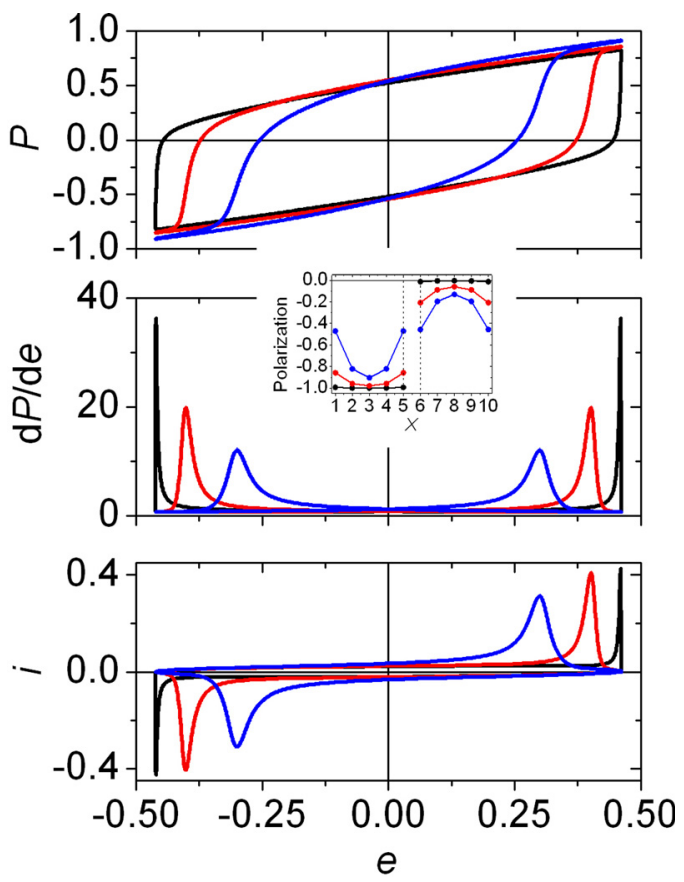

FIG. 4. Influence of interface couplings $\lambda$ on switching characteristics with $e_{0}=1.2 e_{C}$. The value of $\lambda^{-1}$ is: $50(-), 2(-)$, and $0.02(-)$. In the calculations, $\alpha_{1}=-1, \alpha_{2}=1, \beta_{1}=\beta_{2}=1, \kappa_{1}=\kappa_{2}=1$, and $M=N=5$. 
ferroelectric layer and the interface-induced ordered state in the dielectric layer become very strong with $p_{M} \approx q_{1}$ and $p_{1} \approx q_{N}$, if the interface coupling is strong $\lambda^{-1}=0.02$. By increasing the interface coupling, the polarization is enhanced slightly, whereas the coercive field is strongly decreased. The slope of $\mathrm{d} P / \mathrm{d} e-e$ increases and the maximum value of switching current $i_{\max }$ and $\mathrm{d} P / \mathrm{d} e-e$ decreases, as $\lambda$ increases.

In Fig. 5, we examine how the interactions between polarizations within the ferroelectric layer $\kappa_{1}$ and between the induced-polarizations within the dielectric layer $\kappa_{2}$ affect the switching characteristics. The $P$-e hysteresis loop indicates that the polarization decreases and the coercive field increases, as the interaction coefficient $\kappa_{1}$ or $\kappa_{2}$ decreases. As the interaction coefficient $\kappa_{1}$ or $\kappa_{2}$ decreases, the maximum value of $\mathrm{d} P / \mathrm{d} e-e$ increases and the slope of $\mathrm{d} P / \mathrm{d} e-e$ decreases. From the $i$-e curves, the maximum value of $i$ increases with decreasing $\kappa_{1}$ or $\kappa_{2}$.

The properties of ferroelectric materials are expected to change, if the thickness approaches its characteristic length. ${ }^{28}$ In Fig. 6, we show the effect of thickness on the $P$ - $e$ hysteresis loops, $\mathrm{d} P / \mathrm{d} e-e$ and $i-e$ curves for superlattices with different thickness configurations: "10-2," "3-2," and "3-5." We first examine the thickness effect of ferroelectric layer on the switching characteristics of the superlattice, i.e., "10-2" and "3-2" superlattices. Here, the thickness of the dielectric layer is set as $N=2=2 K_{1}^{-1}$. For the superlattice with $M=10>2 K_{1}^{-1}$, the ferroelectric layer is thick enough to sustain a strong ferroelectric behavior, whereas the thickness of the ferroelectric layer approaches the characteristic length, if $M=3 \sim 2 K_{1}^{-1}$. As the $M$ reduces from 10 to 3, the polarization reduces. The polarization is further decreased, if the dielectric layer thickness $N$ is increased from 2 to 5, i.e., the
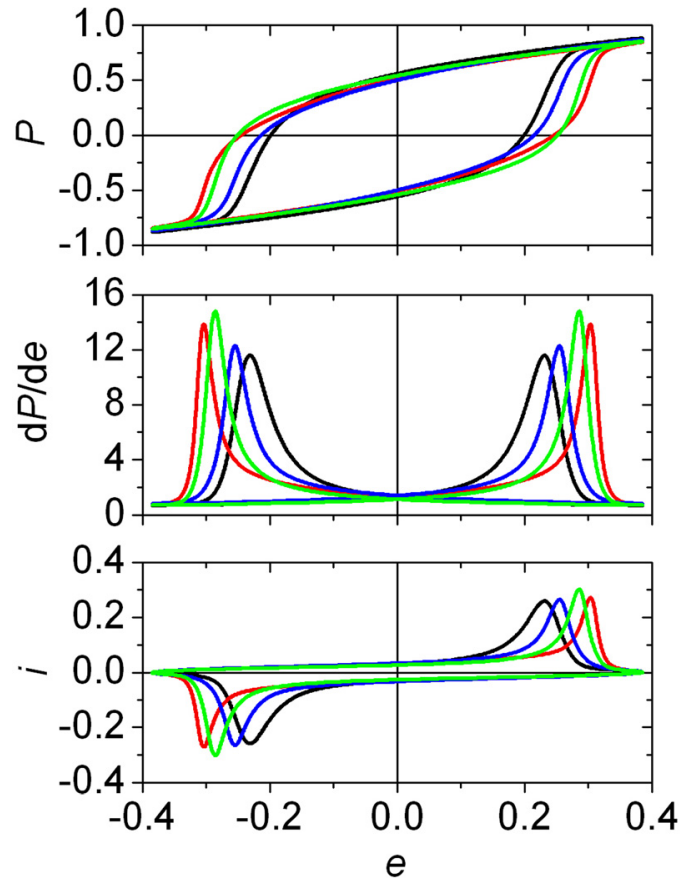

FIG. 5. Influence of interaction coefficients on switching characteristics with $e_{0}=e_{C}$. The values of $\kappa_{1}$ and $\kappa_{2}$ are: 20 and $1(-), 0.5$ and $1(-), 1$ and $20(-)$, and 1 and $0.5(-)$, respectively. In the calculations, $\alpha_{1}=-1, \alpha_{2}=1$, $\beta_{1}=\beta_{2}=1, \gamma_{1}=\gamma_{2}=1, \lambda^{-1}=0.02$, and $M=N=5$.
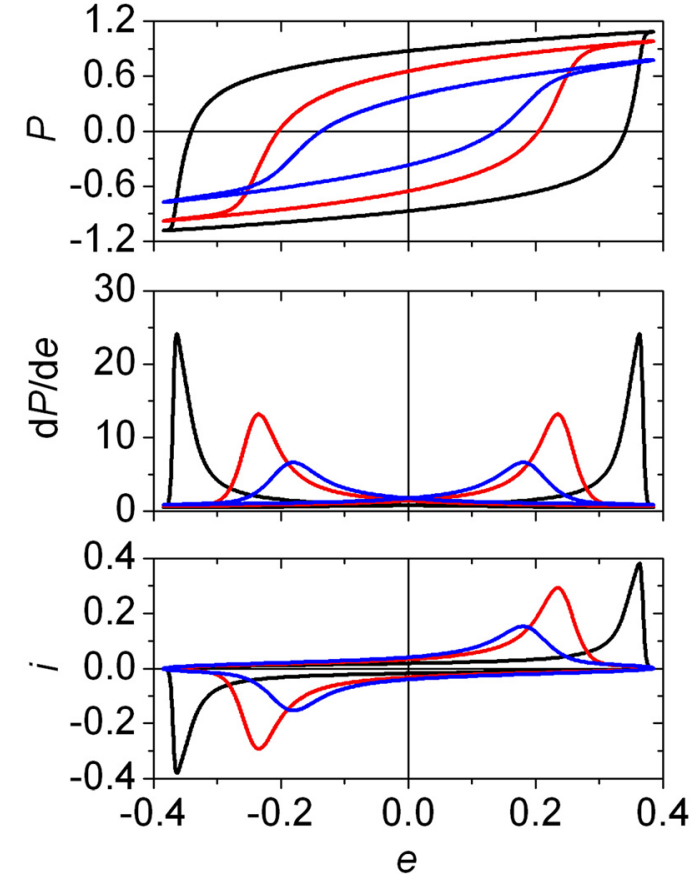

FIG. 6. Influence of layer thickness on switching characteristics with $e_{0}=e_{C}$. The values of $M$ and $N$ are: 10 and $2(-), 3$ and $2(-)$, and 3 and 5 $(-)$, respectively. In the calculation, $\alpha_{1}=-1, \alpha_{2}=1, \beta_{1}=\beta_{2}=1$, $\kappa_{1}=\kappa_{2}=1, \gamma_{1}=\gamma_{2}=1$, and $\lambda^{-1}=0.02$.

"3-5" superlattice. The suppression of polarization as a result of the thickness effect leads to a reduction in the coercive field, the peak value of $\mathrm{d} P / \mathrm{d} e$ and the maximum value of $i$.

Figure 7 illustrates the influence of dielectric stiffness $\alpha_{1}$ on the switching characteristics of a strongly coupled " $3-2$ " superlattice. If $\alpha_{1}=0.02$, the dielectric layer is very soft.
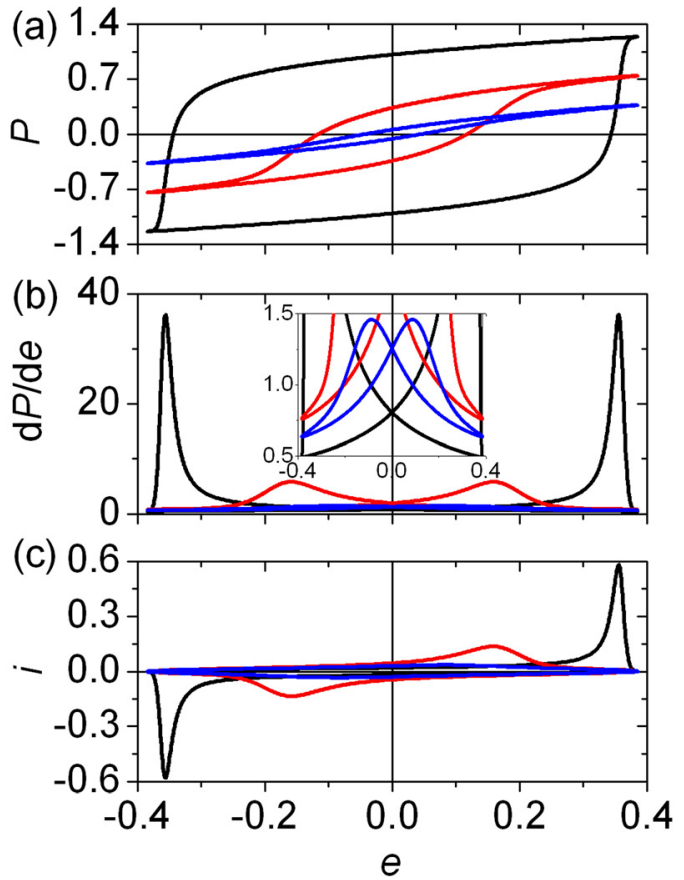

FIG. 7. Influence of dielectric stiffness $\alpha_{2}$ dependent of switching characteristics with $e_{0}=e_{C}, M=3$, and $N=2$. The value of $\alpha_{2}$ is: $0.02(-), 2(-)$, and 5 (-). In the calculations, $\alpha_{1}=-1, \beta_{1}=\beta_{2}=1, \kappa_{1}=\kappa_{2}=1, \gamma_{1}=\gamma_{2}=1$, $\lambda^{-1}=0.02$, and $M=N=5$. 
Thus, the polarization is strongly induced in the dielectric layer via a strong interface coupling. Thus, the polarization and coercive field are strong $\alpha_{1}=0.02$. As the dielectric layer becomes more rigid, the polarization and coercive field decrease. The peak value of $\mathrm{d} P / \mathrm{d} e$ and the maximum value of $i$ decrease, as $\alpha_{1}$ increases.

\section{CONCLUSION}

In this work, we have carried out a systematic investigation on the influence of various model parameters included the field amplitude, viscosity, interface coupling, interaction between neighboring polarizations within the layer, layer thickness and dielectric stiffness on switching characteristics of a superlattice. Our results reveal that the $P$-e hysteresis loop, $\mathrm{d} P / \mathrm{d} e-e$ differential electric susceptibility versus applied field and $i$-e switching current versus applied field depend sensitively on those parameters. The work may provide useful information for the design of ferroelectric memory with specific hysteresis loop by manipulating those parameters in superlattice structures.

\section{ACKNOWLEDGMENTS}

This research was conducted with the support of a University Malaya Research Grant (UMRG-Project No. RP001B-13ICT)/Computation \& Informatics $(\mathrm{C}+\mathrm{i})$ Research Cluster/High-Performance Scientific Computing Program from the University of Malaya (UM). K.H.C. thanks the financial support from the UMRG Grant (No. RP006B-13AFR) from University of Malaya. O.L.H. also acknowledges the supports of RU Grant (1001/PFIZIK/811171) from Universiti Sains Malaysia.

${ }^{1}$ J. F. Scott, "Prospects for Ferroelectrics: 2012-2022," ISRN Mater. Sci. 2013, 24 (2013).

${ }^{2}$ J. F. Scott, Ferroelectric Memories (Springer, Verlag, 2000).

${ }^{3}$ M. Dawber, C. Lichtensteiger, M. Cantoni, M. Veithen, P. Ghosez, K. Johnston, K. M. Rabe, and J.-M. Triscone, Phys. Rev. Lett. 95, 177601 (2005).

${ }^{4}$ E. D. Specht, H. M. Christen, D. P. Norton, and L. A. Boatner, Phys. Rev. Lett. 80, 4317 (1998)

${ }^{5}$ J. Sigman, D. P. Norton, H. M. Christen, P. H. Fleming, and L. A. Boatner, Phys. Rev. Lett. 88, 097601 (2002).

${ }^{6}$ H. Tabata, H. Tanaka, and T. Kawai, Appl. Phys. Lett. 65, 1970 (1994).

${ }^{7}$ S. Ríos, A. Ruediger, A. Q. Jiang, J. F. Scott, H. Lu, and Z. Chen, J. Phys.: Condens. Matter 15, L305 (2003).

${ }^{8}$ N. Ortega, A. Kumar, O. A. Maslova, Yu. I. Yuzyuk, J. F. Scott, and R. S. Katiyar, Phys. Rev. B 83, 144108 (2011).

${ }^{9}$ R. Ranjit, B. Kundys, and W. Prellier, Appl. Phys. Lett. 91, 222904 (2007).
${ }^{10}$ P. Zubko, N. Jecklin, A. Torres-Pardo, P. Aguado-Puente, A. Gloter, C. Lichtensteiger, J. Junquera, O. Stéphan, and J.-M. Triscone, Nano Lett. 12, 2846 (2012).

${ }^{11}$ D. D. Fong, C. Cionca, Y. Yacoby, G. B. Stephenson, J. A. Eastman, P. H. Fuoss, S. K. Streiffer, C. Thompson, R. Clarke, R. Pindak, and E. A. Stern, Phys. Rev. B 71, 144112 (2005).

${ }^{12}$ J. Shin, A. Y. Borisevich, V. Meunier, J. Zhou, E. W. Plummer, S. V. Kalinin, and A. P. Baddorf, ACS Nano 4, 4190 (2010).

${ }^{13}$ C.-L. Hung, Y.-L. Chueh, T.-B. Wu, and L.-J. Chou, J. Appl. Phys. 97, 034105 (2005).

${ }^{14}$ Y. Ishibashi, N. Ohashi, and T. Tsurumi, Jpn. J. Appl. Phys., Part 1 39, 186 (2000).

${ }^{15}$ V. R. Cooper, K. Johnston, and K. Rabe, Phys. Rev. B 76, 020103R (2007).

${ }^{16}$ N. A. Pertsev and M. Tyunina, J. Appl. Phys. 109, 126101 (2011).

${ }^{17}$ K.-H. Chew, L.-H. Ong, J. Osman, and D. R. Tilley, Appl. Phys. Lett. 77, 2755 (2000).

${ }^{18}$ L.-H. Ong, J. Osman, and D. R. Tilley, Phys. Rev. B 65, 134108 (2002).

${ }^{19}$ B. Yang, D.-M. Zhang, C.-D. Zheng, J. Wang, and J. Yu, J. Phys. D: Appl. Phys. 40, 5696 (2007).

${ }^{20}$ C.-D. Zheng, D.-M. Zhang, X.-M. Liu, B. Yang, C.-J. Liu, and J. Yu, Chin. Phys. Lett. 27, 017702 (2010).

${ }^{21}$ L.-H. Ong, T.-Y. Lee, and K.-H. Chew, Ceram. Int. 38, S3 (2012).

${ }^{22}$ Y. Y. Liu and J. Y. Li, Appl. Phys. Lett. 97, 042905 (2012).

${ }^{23}$ Y. Ishibashi, Integr. Ferroelectr. 2, 41 (1992).

${ }^{24}$ Y. Ishibashi, J. Phys. Soc. Jpn. 59, 4148 (1990).

${ }^{25}$ M. Omura, H. Adachi, and Y. Ishibashi, Jpn. J. Appl. Phys., Part 1 30, 2384 (1991).

${ }^{26}$ M. Omura, H. Adachi, and Y. Ishibashi, Jpn. J. Appl. Phys., Part 1 31, 3238 (1992).

${ }^{27}$ M. Omura, T. Ishibahi, and Y. Ishibashi, Jpn. J. Appl. Phys., Part 1 32, 4388 (1993).

${ }^{28}$ K.-H. Chew, Y. Ishibashi, and F. G. Shin, J. Phys. Soc. Jpn. 75, 064712 (2006).

${ }^{29}$ K.-H. Chew, L.-H. Ong, and M. Iwata, J. Appl. Phys. 110, 054108 (2011).

${ }^{30}$ K.-H. Chew, Solid State Phenom. 189, 145 (2012).

${ }^{31}$ K.-G. Lim, K.-H. Chew, L.-H. Ong, and M. Iwata, EPL 99, 46004 (2012).

${ }^{32}$ K.-H. Chew, Y. Ishibashi, F. G. Shin, and H. L.W. Chan, J. Phys. Soc. Jpn. 72, 2364 (2003).

${ }^{33}$ C. H. Tsang, K.-H. Chew, Y. Ishibashi, and F. G. Shin, J. Phys. Soc. Jpn. 73, 3158 (2004).

${ }^{34}$ K.-H. Chew, L.-H. Ong, and M. Iwata, Curr. Appl. Phys. 11, 755 (2011).

${ }^{35}$ Y. Zhou, Solid State Commun. 150, 1382 (2010).

${ }^{36}$ D. Ricinschi, Y. Ishibashi, M. Iwata, and M. Okuyama, Jpn. J. Appl. Phys., Part 1 40, 4990 (2001).

${ }^{37}$ L. Baudry and J. Tournier, J. Appl. Phys. 90, 1442 (2001).

${ }^{38}$ D. Ricinschi, A. I. Lerescu, and M. Okuyama, Jpn. J. Appl. Phys., Part 1 39, L990 (2000).

${ }^{39}$ M. Stengel and N. A. Spaldin, Nature 443, 679 (2006).

${ }^{40}$ M. Stengel, D. Vanderbilt, and N. A. Spaldin, Nature Mater. 8, 392 (2009).

${ }^{41}$ L.-W. Chang, M. Alexe, J. F. Scott, and J. M. Gregg, Adv. Mater. 21, 4911 (2009).

${ }^{42}$ B. D. Qu, W. L. Zhong, and R. H. Prince, Phys. Rev. B 55, 11218 (1997).

${ }^{43}$ W. H. Press, S. A. Teulosky, W. T. Vetterling, and B. P. Flannery, Numerical Recipes in Fortran 77: The Art of Scientific Computing, 2nd ed. (Cambridge University Press, Cambridge, 2001), Vol. 1.

${ }^{44}$ K.-H. Chew, Y. Ishibashi, and F. G. Shin, J. Phys. Soc. Jpn. 74, 2338 (2005). 